INPLASY

PROTOCOL

To cite: Wang et al. The Effect of Massage and Myofascial Release on the Pain Degree of Patients with Chronic Neck Pain: A Systematic Review and Meta-analysis. Inplasy protocol 202180064. doi:

10.37766/inplasy2021.8.0064

Received: 15 August 2021

Published: 15 August 2021

Corresponding author:

Qi Gao

fevok@sina.com

Author Affiliation:

Beijing Sport University

Support: Beijing Sport

University.

Review Stage at time of this submission: Preliminary searches.

Conflicts of interest:

None declared.

\section{The Effect of Massage and Myofascial Release on the Pain Degree of Patients with Chronic Neck Pain: A Systematic Review and Meta-analysis}

Wang, S1; Jiang, A2; Gao, Q33.

Review question / Objective: The purpose of this study is systematic evaluate whether massage and myofascial release can effectively alleviate the degree of neck pain in patients with chronic neck pain.

Condition being studied: Chronic neck pain is currently the world's second largest health hazard after chronic low back pain. With the acceleration of the social rhythm and changes in the living and working environment, the number of cases of neck pain is increasing year by year. The main clinical manifestations of patients with neck pain include neck pain, decreased range of motion, weakness or fatigue of surrounding muscles, abnormal breathing patterns, and impaired proprioception.

INPLASY registration number: This protocol was registered with the International Platform of Registered Systematic Review and Meta-Analysis Protocols (INPLASY) on 15 August 2021 and was last updated on 15 August 2021 (registration number INPLASY202180064).

\section{INTRODUCTION}

Review question / Objective: The purpose of this study is systematic evaluate whether massage and myofascial release can effectively alleviate the degree of neck pain in patients with chronic neck pain.
Condition being studied: Chronic neck pain is currently the world's second largest health hazard after chronic low back pain. With the acceleration of the social rhythm and changes in the living and working environment, the number of cases of neck 
pain is increasing year by year. The main clinical manifestations of patients with neck pain include neck pain, decreased range of motion, weakness or fatigue of surrounding muscles, abnormal breathing patterns, and impaired proprioception.

\section{METHODS}

Participant or population: Chronic neck pain patients refer to people whose neck pain lasts for more than one month (previously three months or six months). Exclude patients with neck pain caused by trauma and patients with rheumatic immunity or radiculopathy.

Intervention: Massage is a method of manipulating specific parts of the body surface to adjust the physiological and pathological conditions of the body to achieve the purpose of physical therapy. Myofascial release is a treatment method for loosening adhesion of human muscle fascia.

Comparator: The control group was blank control, placebo, exercise or physical therapy.

Study designs to be included: Randomized controlled trials are eligible for inclusion in the review.

Eligibility criteria: The type of literature research was randomized controlled trials. Experimental group received massage or myofascial release; control group was blank control, placebo, exercise or physical therapy. Measurements and results were pain level evaluation scales such as visual analogue scale (VAS), 11-point numerical pain rating scale, neck pain questionnaire.

Information sources: Electronic databases: PubMed, EMBASE, Cochrane Library, Web of Science. The retrieval date was January 1, 2000. to August 10, 2021.

Main outcome(s): The main outcome indicator is pain level evaluation scale such as visual analogue scale (VAS), 11-point numerical pain rating scale, neck pain questionnaire.
Quality assessment / Risk of bias analysis: The risk of bias and quality of included RCTs were assessed by the Cochrane riskof-bias tool. It includes seven specific domains: random sequence generation, allocation concealment, blinding of participants and personnel, blinding of outcome assessment, incomplete outcome data, selective outcome reporting and other sources of bias.

Strategy of data synthesis: STATA statistical software $\mathbf{1 6 . 0}$ was used for metaanalysis. Standardized mean differences (SMD) and 95\% confidence interval (Cl) were calculated. The heterogeneity among selected studies was assessed with the $\mathbf{I}^{2}$ test. Egger's test and Begg's test were used to assess potential publication bias.

Subgroup analysis: If the heterogeneity is significant, we will conduct a subgroup analysis. The different treatment types and different scales for evaluating pain were classified and compared to determine the source of heterogeneity.

Sensitivity analysis: Sensitivity analysis can make the experimental results more reliable and stable.

\section{Country(ies) involved: China.}

Keywords: Chronic Neck Pain; Myofascial Release; Massage; Manual therapy; Metaanalysis.

Contributions of each author:

Author 1 - Siqi Wang.

Email: wangsiqiace@163.com

Author 2 - Aiyun Jiang.

Email: irene0340@126.com

Author 3 - Qi Gao.

Email: fevok@sina.com 